\title{
An Application of the Miertus-Scrocco-Tomasi Solvation Model in Molecular Mechanics and Dynamics Simulations
}

\author{
A. A. VARNEK ${ }^{\dagger}$ and G. WIPFF* \\ URA 422 CNRS, Institut de Chimie, 4, Rue Blaise Pascal, 67000 Strasbourg, France
}

\author{
A. S. GLEBOV \\ Mendeleev University of Chemical Technology, Miusskaya Square, 9, Moscow, Russia \\ D. FEIL \\ Department of Chemical Technology, University of Twente, Enschede, The Netherlands
}

Received 3 January 1994; accepted 8 June 1994

\begin{abstract}
The point-chart approximation of the Miertus-Scrocco-Tomasi solvation model (MST-PC) based on a continuum representation of the solvent has been incorporated in force field calculations. Application in molecular mechanics (MM) involves conformational equilibria in solution: rotational isomers of ethylene glycol (I), 1,2-difluoroethane (II), fluoroacetic acid (III), and representative conformers of macrocyclic receptors such as 18-crown-6 (IV), cryptand 2.2.2 (V), and $t$-butyl-calix[4]arenetetraamide (VI). Assessment of the MST-PC results is based on the comparison with ab initio reactive field calculations (for I-III), with the continuum model of Still (W. C. Still et al., J. Am. Chem. Soc., 1990, 112, 6127) (for I-VI), and with average solute-solvent interaction energies obtained from molecular dynamics (MD) simulations with explicit solvent in water (I-VI) and in acetonitrile (IV-VI). It is demonstrated that the continuum solvent model qualitatively reproduces the trends in solvation energies in water. The few exceptions may be related to particular topological features of the solute. An improved discrete/continuum approach in which some first-shell solvent molecules are considered as a part of the solute embedded in the dielectric continuum provides more realistic results, as is
\end{abstract}

\footnotetext{
*Author to whom all correspondence should be addressed.

${ }^{\dagger}$ Permanent address: Mendeleev University of Chemical Technology, Miusskaya Square, 9, Moscow, Russia.
} 
shown for VI in water. The MST-PC model which mimics the solute-solvent electrostatic interaction only fails to reproduce conformationally dependent solvation energies in acetonitrile, in which the electrostatic contribution is relatively small compared to van der Waals interactions. Exploratory MD simulations within the continuum model in water are reported on urea and 18-crown- 6 . (c) 1995 by John Wiley \& Sons, Inc.

\section{Introduction}

$\mathrm{n}$ recent years there has been considerable interest in the interpretation and computer representation of solvent effects on the conformational properties of molecules. ${ }^{1-8}$ The methods to study molecular solvation can be broadly classified into those based on discrete or on continuum models of the solvent. In discrete models all solvent molecules are treated explicitly and simulated either by molecular dynamics (MD) or Monte Carlo (MC). These methods provide the microscopic pictures of the solution and a variety of related thermodynamic and kinetic properties. Because they require significant computation times, efforts are made to develop less expensive approaches based on the macroscopic representation of liquids.

The continuum methods consider the interaction of the solute with the solvent represented by a homogeneous dielectric continuum. Among them, the boundary element (BE) methods ${ }^{9}$ have received much attention. They consider the solute embedded inside a cavity in the dielectric continuum. The problem of obtaining the media reactive field is reduced to the calculation of surface charges at the dielectric boundaries. Solvation is treated as an electrostatic interaction between the solute and its polarized cavity. Such a methodology was intensively used in the works of Huron, Claverie et al., ${ }^{10-14}$ Levitt, ${ }^{15}$ Rashin, ${ }^{16-18}$ Zauhar and Morgan, ${ }^{19-21}$ and Vorobjev et al. ${ }^{22}$

The BE approach has a number of advantages compared to the finite difference method (FDM $),{ }^{9,23,24}$ which is widely used to study electrostatics effects in solution within a continuum model. The number of volume elements required in the FDM increases rapidly with the size of the system under investigation, whereas in the $B E$ method the number of elements increases more slowly because only the surface of the solute rather than its volume is being modeled. The reaction field is directly computed in BEM from the distribution of the surface-charge density; it cannot be directly computed in FDM.
At the beginning of the 1980s, Miertus, Scrocco, and Tomasi (MST) elaborated a version of the BE model $^{25,26}$ in which the effective solvent operator is defined in such a way as to permit a direct representation of the multipole-multipole interactions to all orders. It uses cavities of any shape, defined as an ensemble of interlocking spheres centered on the atoms of the solute. This model was originally designed within the framework of RHF-SCF-MO-LCAO nonempirical quantum mechanical calculations (MST-QM). The numerous applications of the MST-QM approach concern absorption $^{27}$ and vibrational ${ }^{28}$ spectra, reactivity, ${ }^{29-31}$ and conformational stability ${ }^{32}$ of the solutes in pure solvents. Tomasi et al. ${ }^{33}$ extended that method for two-phase liquid-liquid systems and for anisotropic solvents. An incorporation of the MST approach into semiempirical MNDO and AM1 quantum mechanical calculations has been recently reported. ${ }^{34-36}$ Other versions of the continuum model were effectively used in quantum mechanical calculations of Rivail, ${ }^{37,38}$ Truhlar, $^{39}$ and others (see ref. 40 for a review). These models do not take into account solvent-solvent interactions explicitly but represent the solute-solvent electrostatic interactions.

The application of the MST-QM to calculate molecular geometry in solution ${ }^{41}$ is limited by the number of atoms of the solute. Therefore, attempts have been made to couple a classical part of the MST method with a force field representation of the energy of the solute. This can be achieved by simplifying the calculation of the solute-solvent interaction energy for applications in the molecular mechanics, molecular dynamics, or Monte Carlo simulations.

Recently the MST method in the point-charge approximation (MST-PC) was used to calculate solvation energies of rotational isomers of 1,2ethanediol $^{42}$ and different conformers of 1,2ethanediol diformate. ${ }^{31,43}$ In ref. 43 , the conformational energy of the solute was computed using the MM2 force field ${ }^{44}$ without energy minimization, and the MST-PC solvation energies were compared with MST-QM values.

Some recent applications in MM, MC, and MD of continuum models different from the MST one 
should be noted. Allinger et al. ${ }^{45,46}$ proposed the "Induced Dipole Moment and Energy" method (coupled with the MM2 program ${ }^{44}$ ) to calculate the solvation energies of neutral solutes in spherical cavities. Sklenar et al. ${ }^{6}$ have developed the FIESTA method, which calculates solvation energies of charged solutes taking into account counterion effects. In contrast to finite difference method, ${ }^{9,24}$ the integration grid in FIESTA is associated with atomic volumes. Soumpasis ${ }^{47,48}$ incorporated in the AMBER software an analytical approximation for the potential of mean force for treating ionic effects of biomolecular structures in solution. Westhof and Fritsch ${ }^{49,50}$ added to AMBER the sigmoidal dielectric function suggested by Lavery ${ }^{51}$ to perform MD simulations of nucleic acids. Zauhar incorporated the polarization-charge technique into the CHARM package ${ }^{52}$ and used it for a MM calculation of a tripeptide. ${ }^{53}$ This approach uses cavities of arbitrary shape and harmonic constrains to mimic solvation forces. Beveridge et $\mathrm{al}^{54-56}$ used a combined discrete/continuum model in MC simulations of ion hydration.

Efforts have been made recently to use the continuum model in molecular dynamics. Thus, Sharp ${ }^{57}$ has incorporated solvent effects into the MD procedure using finite difference method. ${ }^{9}$ To our knowledge, there are no publications concerning the application of the $\mathrm{BE}$ approach in $\mathrm{MD}$ simulations.

Recently Still et al. ${ }^{58,59}$ developed the GB/SA, method in which the solvation energy is calculated as a sum of a term which corresponds to van der Waals and cavitation energies (proportional to solvent accessible "SA" surface) and a solute-solvent electrostatic polarization term (written in the form of a generalized Born "GB" equation). This approach was incorporated in the MacroModel program. ${ }^{60}$

In this article we report several tests of the MST-PC approach incorporated in MM or MD to calculate geometries and energy parameters of molecules in solution. We focus on the following questions:

1. Does the point-charge approximation reproduce trends in solvation energies as obtained from MST-QM calculations?

2. The MST model pictures only the electrostatic component of the solvent-solute interaction energy. However, do MST-PC solvation energies correlate with total solute-solvent interaction energies obtained after MD or MC simulations with explicit representation of a solvent, where Coulomb and van der Waals components are calculated explicitly?

3. Until now, most of the calculations with the MST model were done for aqueous solutions. It is not clear if this method gives reliable results for nonaqueous solvents. Therefore, we considered solvation in water and in acetonitrile solutions.

4. Can we improve the pure MST model by explicit consideration of the most significant first-shell solvent molecules? Indeed, the specific solvation pattern, such as hydrogen bonding, has been shown to bring a major contribution to the solvation energy but is neglected in the pure MST model. Taking advantage of both the continuum model and the force field methodology, we propose a combined discrete/continuum approach, in which the first solvation shell is modeled explicitly and is embedded with the solute in the cavity.

5. To what extent is the structure of a solute affected by solvent? A comparison of structures of a molecule in vacuo and in solution might shed light on the influence of solvent effects on the molecular conformations.

In this article we focus on the solvent--solute interactions rather than enthalpy or free energy of solvation, which include solvent--solvent interactions.

We have performed MM calculations based on the MST-PC model on different conformers of ethylene glycol (I), 1,2-difluoroethane (II), fluoroacetic acid (III), 18-crown-6 (IV), cryptand 2.2.2 (V), and $t$-butyl-calix[4]arenetetraamide (VI) to study the conformational equilibria of compounds with different topology in aqueous and in acetonitrile solutions.
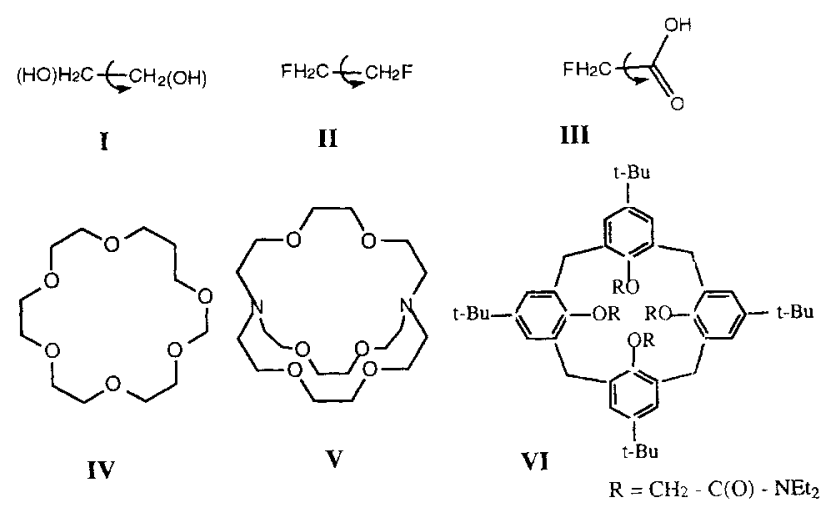
Molecules I-III are small enough to perform test calculations by the MST-QM method and MD simulations in a bath of explicit solvent molecules. Solvation of IV-VI in water and in acetonitrile has been explicitly studied by MD and MC simulations. ${ }^{4,61-63}$ To investigate the hydration of some conformers of 18 -crown- 6 , several MD simulations in water have been rerun in this work.

We investigated the influence of empirical parameters of the MST-PC method on the solvation energies by performing MM calculations on phosphoric acid in tetrahydrofuran (THF) solution.

The possibility of applying the MST-PC model in MD simulations is discussed on the basis of the results obtained for urea and 18-crown-6 in water.

Finally, we have performed calculations on I-VI by the GB/SA method ${ }^{60}$ to compare hydration energies obtained in different continuum models.

\section{The MST Model in Ab Initio and Point-Charge Approximations}

\section{CALCULATION OF THE SOLVATION ENERGY}

The solute (M), represented by interlocking spheres centered on the atoms of $\mathbf{M}$, is embedded into a cavity in a homogeneous dielectric continuum. In the original version (MST-QM), ${ }^{25,26}$ the solute-solvent interaction is described via the Hartree-Fock equations, whose Hamiltonian $H=$ $H^{0}+V_{\sigma}$ includes both vacuo $\left(H^{0}\right)$ and solvent $\left(V_{\sigma}\right)$ contributions.

The effective solvation potential $V_{\sigma}$ is defined there is a semiclassical approximation (i.e., taking into account only the Coulombic and polarization terms of the solute-solvent interaction and representing the solvent as a continuum with a dielectric permittivity $\varepsilon$ ). $V_{\sigma}$ is obtained from the charge distribution $\sigma(s)$ over the surface of the cavity. The relationship for calculation of $\sigma(s)$ is

$$
\sigma(s)=\frac{\varepsilon-1}{4 \pi \varepsilon} \frac{\partial}{\partial n}\left(V_{\chi}+V_{\sigma}\right)_{s}
$$

where the derivative of the potential is computed along the inner normal to the cavity. At the point $s$, the two components $V_{\chi}$ and $V_{\sigma}$ of the electrostatic potential correspond, respectively, to the charge distribution $\chi_{M}(r)$ of the solute and to the cavity charge distribution $\sigma(s)$. The latter is calculated by an iterative self-polarization procedure putting $V_{\sigma}=0$ for zero approximation to obtain $\sigma^{0}(s)$. Then, $V_{\sigma}$ and $\sigma(s)$ are calculated iteratively until consistency is reached.

The calculations performed for a limited number of points $s$ lead to point charges $q_{k}$ placed at the center of $k$ th cavity fragment of area $\Delta S_{k}$ :

$$
q_{k}=\sigma(s) \Delta S_{k}
$$

Finally, the effective electrostatic potential is

$$
V_{\sigma}(r)=\sum q_{k} /\left|r-S_{k}\right|
$$

The free energy of solvation $\Delta F_{\text {solv }}$ is estimated as the electrostatic interaction energy between the solute and the polarized cavity:

$$
\Delta F_{\mathrm{solv}}=\left(E-E^{0}\right)-\frac{1}{2} \int \chi_{M}(r) V_{\sigma}(r) d r
$$

where the total energies $E^{0}$ and $E$ correspond, respectively, to the Hamiltonians $H^{0}$ and $H$.

It was emphasised in ref. 26 that evaluation of $\Delta F_{\text {solv }}$ does not actually require quantum mechanical calculations. Equation (4) may be simplified by the following approximations: (1) the neglect of the effects of polarization of the solute in the solvent reaction field; and (2) the representation of the molecular charge density $\chi_{M}(r)$ by a set of atomic charges $Q_{i}$. In this case, $\Delta F_{\text {solv }}$ may be calculated as ${ }^{25,26}$

$$
\Delta F_{\text {solv }}=\frac{1}{2} \sum Q_{i} V_{\sigma, i}
$$

where $V_{\sigma, i}$ is the effective solvent potential calculated at the position of $i$ th atomic charge.

A linear correlation has been observed between the $\Delta F_{\text {solv }}$ values calculated by eq. (4) and those calculated by eq. (5) for 19 small molecules ${ }^{31}$ and for several conformers of 1,2-ethanediol diformate $^{43}$ This means that the MST-PC point-charge approximation reproduces the same trends in solvation energies as do quantum mechanical calculations.

Alagona and Ghio $^{42}$ and Tomasi ${ }^{31}$ have shown that an additional coefficient $f$ should be introduced to scale MST-PC solvation energies to MSTQM ones. In our calculations we used a scaling factor $f=2$. This value is intermediate between $f=1.1$ (obtained for rotational isomers of 1,2ethanediol calculated with 4-31G and 6-31G* basis sets $^{42}$ ) and $f \approx 4$ for 19 small molecules (calculated with $6-31 \mathrm{G}^{* *}$ basis set $\left.^{31}\right)$ ]. Claverie et al. ${ }^{64}$ used $f=0.63$ to reproduce the experimental va- 
porization energy of water within the continuum model.

An empirical proportionality has been observed between the free energy of solvation $\Delta F_{\text {solv }}$ and its enthalpic component $\left(E_{\text {solv }}\right){ }^{26}$ The former quantity relates to the MST-PC model whereas $E_{\text {solv }}$ relates to the MD simulations with explicit solvent, from which the average solute-solvent interaction energy is extracted. An application of appropriate factor $f$ in calculations of $E_{\text {solv }}$ [eq. 6] might bring those energies on the same scale.

$$
E_{\mathrm{solv}}=f\left(\frac{1}{2} \sum Q_{i} V_{\sigma, i}\right)
$$

In the MD simulations, solvation forces on atoms (or energy derivatives in $\mathrm{MM}$ ) due to the tesserae charges were evaluated analytically according to the Coulomb law. We did not take into account the solvent pressure forces, ${ }^{24,53,65}$ which are more important for charged and flexible solutes than for the neutral and relatively rigid molecules considered here.

\section{DETERMINATION OF THE SHAPE AND SIZE OF THE CAVITY}

The cavity is defined by the ensemble of intersecting spheres centered on the atoms of the solute. ${ }^{66}$ Each sphere is represented by the pentakisdodecahedron with 60 triangle faces (tesserae). When two spheres intersect, the triangles of the first one situated inside the second one are eliminated. To obtain an adequate description of the tesserae at intersections, a further tesserae partition is performed (see Fig. 2 in ref. 66). At each partition level (defined by the parameter ND), each triangular tessera is divided into four equivalent triangles. For example, for the first level the original tessera is not divided; for the fifth level $(N D=5)$, it is divided into $4^{4}=256$ triangles. The surface is described by a number of such triangles, which are then grouped into a unique patch. The van der Waals radii of the solute atoms were scaled by 1.2 to obtain optimal cavity. ${ }^{26,36}$

To describe the solvent-excluded volume, the program creates iteratively additional spheres, which are then treated as the original ones, until the radius of the additional sphere is less than $R_{\min }$ (see Fig. 5 in ref. 66). The calculated molecular surface is thus a function of the sizes of the solute atoms and of the effective diameter of solvent. Other techniques ${ }^{21,53,67}$ to calculate the shape and size of the cavity have been implemented in $\mathrm{BE}$ methods.

\section{Computation Procedure}

\section{SOFTWARE FOR MOLECULAR MECHANICS CALCULATIONS WI'TH THE MST-PC APPROACH INCORPORATED}

The program package MM2IS ${ }^{68}$ performs molecular mechanics calculations of rather complex systems (molecules, associates, complexes, etc.) in solution using a continuum or discrete/continuum representation of the solvent. The total energy of the solute is presented as a sum of intrinsic $\left(E_{\text {intra }}\right)$, intermolecular $\left(E_{\text {inter }}\right)$, and solvation $\left(E_{\text {solv }}\right)$ terms.

$E_{\text {intra }}$ calculated using the MM2 force field ${ }^{44}$ includes deformation energies of bonds and angles and dihedral and noncovalent interactions within the solute. $E_{\text {inter }}$ describes noncovalent interactions within the first solvation shell of the solute with a modified $^{69}$ Claverie force field ${ }^{70}$ as a sum of electrostatic $\left(E_{\mathrm{es}}\right)$, polarization $\left(E_{\mathrm{pol}}\right)$, and van der Waals $\left(E_{\mathrm{VDW}}\right)$ terms:

$$
E_{\text {inter }}=E_{\mathrm{es}}+E_{\mathrm{pol}}+E_{\mathrm{VDW}}
$$

$E_{\mathrm{es}}$ is calculated in the point-charge approximation. $E_{\mathrm{pol}}$ is computed ${ }^{71}$ as follows:

$$
E_{\mathrm{pol}}=-\frac{1}{2} \sum \alpha_{j} F_{j}^{2}
$$

where $F_{j}$ is the electric field at the atom $j$, and atomic polarizabilities $\alpha_{j}$ are calculated using an additive scheme proposed in ref. 71 from experimental data on bond polarizabilities. ${ }^{72} E_{\mathrm{VDW}}$ is calculated by

$$
\begin{aligned}
E_{\mathrm{VDW}}=\sum \sum k_{i j}[ & -A / Z_{i j}^{6}+B_{0}\left(1-Q_{i} / N_{i}\right) \\
& \left.\times\left(1-Q_{j} / N_{j}\right) \exp \left(-b Z_{i j}\right)\right]
\end{aligned}
$$

where $Z_{i j}=R_{i j} /\left(R_{i}^{*} R_{j}^{*}\right)^{1 / 2} ; R_{i j}$ is the interatomic distance; $N_{j}$ is the number of valence electrons; $Q_{j}$ is the atomic charge; $R_{j}^{*}$ is the van der Waals radius of atom $j$; and $A, b$, and $B_{0}$ are fixed parameters defined in ref. 70 .

The gas-phase calculations of the crown ethers, phosphorus-containing mono- and polypodands, and their complexes with alkali cation and ion pair aggregates ${ }^{69,73-77}$ showed the reliability of the combined MM2 + Claverie force field, thus allowing its application to related complex molecular systems. 
The solvation energy $E_{\text {solv }}$ is calculated in terms of the MST-PC approach according to eqs. (1), (2), (3), and (6). Some subroutines from the MGPISA software $^{78}$ are used in MM2IS to calculate the geometry of the cavity and charges on the tesserae. All force field parameters are stored in the DATA file and can be easily modified. We used the dielectric permittivities $78.5,37.5$, and 7.58 and densities 1.0, 0.777, and $0.884 \mathrm{~g} / \mathrm{mL}$ for water, acetonitrile, and tetrahydrofuran (THF), respectively. ${ }^{79}$ The MD_DRAW software ${ }^{80}$ has been used to visualize the molecular surface of the solutes. Atomic electrostatic potential (ESP) charges ${ }^{81}$ for I-III (Chart I) were obtained by the least-squares method fitting the MNDO molecular electrostatic potential ${ }^{82}$ at the Connolly surfaces.$^{83}$ ESP charges for IV, V, and VI were taken from refs. 61, 84, and 62 , respectively. These sets of charges were also used in the GB/SA calculations of I-VI and MD simulations with AMBER on I-IV in the box of explicit water. A series of the MST-PC calculations was performed on IV with the charges from ref. 69 to elucidate to what extent the charge distribution
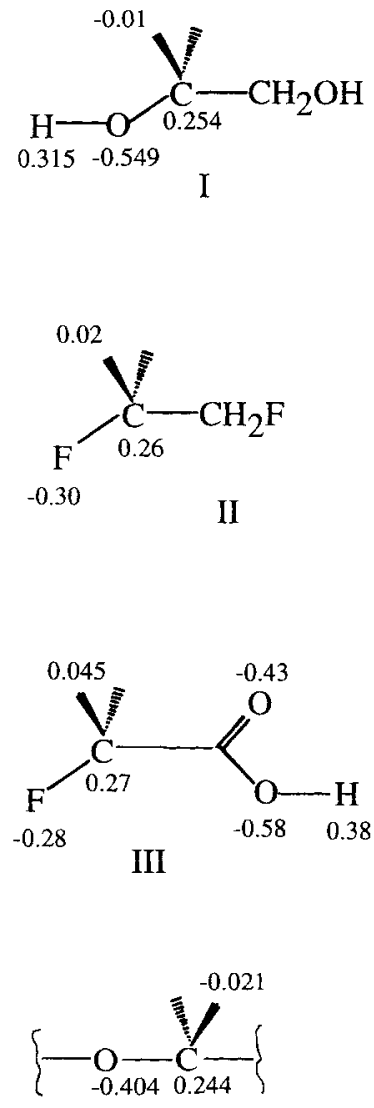

IV
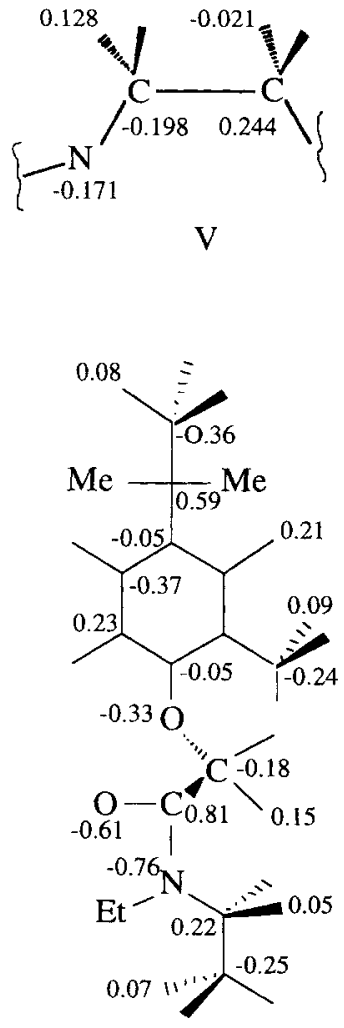

VI
CHART I. Charge distribution in I-VI. of a solute may influence relative solvation energies. The MST-PC calculations were performed on rigid solutes (I-VI). A series of calculations was also performed on I and IV with the geometry optimization of the solute.

\section{SOFTWARE FOR MOLECULAR DYNAMICS SIMULATIONS WITH THE MST-PC APPROACH INCORPORATED}

The GROMOS software ${ }^{85}$ has been modified $^{86}$ to perform MD simulations of molecules situated inside the cavity in a polarized continuum. The subroutines for the calculations of size and shape of the cavity, of the charges on the tesserae, of the forces, and of the solvation energies, which are similar to those in MM2IS, are called from the subroutine FORCE.

The standard parameters of the GROMOS force field $^{87}$ were used for urea. 18-Crown-6 was treated in an all-atom model using GROMOS van der Waals and covalent parameters. ${ }^{87}$ The atomic charges from ref. 69 were used for calculations of intramolecular and solute-cavity electrostatic energies.

Covalent bonds involving hydrogen atoms were constrained by SHAKE, allowing a timestep of 2 fs. Energy minimization of the system was followed by $40-80 \mathrm{ps}$ of MD. The temperature was maintained at $300 \mathrm{~K}$ by velocity scaling using a relaxation time of $0.1 \mathrm{ps}$.

\section{MOLECULAR DYNAMICS SIMULATIONS WITH EXPLICIT SOLVENT}

Unless otherwise specified, the MD results in explicit water and acetonitrile solutions come from previous work on crown ether IV, ${ }^{63,88}$ cryptand V, ${ }^{63,89}$ and calixarene VI.,62 For rigid solutes I-IV, an additional 50 ps of MD simulations were performed in a box of about 200 TIP3P $^{90}$ water molecules at $300 \mathrm{~K}$ and $1 \mathrm{~atm}$ with AMBER4. ${ }^{91,92}$

\section{MST-QM CALCULATIONS}

These calculations were performed on the syn, gauche, and anti conformers of I-III with the STO-3G basis set using the MGPISA software. ${ }^{78}$ Standard values of bond lengths and valence angles were used for the starting structures. ${ }^{93}$

\section{GB / SA CALCULATIONS}

These calculations were performed on I-VI using the MacroModel software ${ }^{60}$ with the same 
charges and starting geometries of the solutes as in the MST-PC calculations.

\section{Results and Discussion}

\section{MOLECULAR MECHANICS WITH THE MST-PC METHOI) INCORPORATED}

\section{Continuum Representation of a Solvent}

Influence of the Parameters of the MST-PC Model on the Solvation Energies Calculations. The calculated cavity shape and tesserae charges depend on the following parameters: ND (the level of intersected tesserae division), $R_{\text {min }}$ (the minimal radius of sphere to be created by the program), and $N L$ (the number of loops of self-polarization). The data collected in Tables I through III for phosphoric acid $\mathrm{PO}(\mathrm{OH})_{3}$ in THF and 18-crown-6 $\left(\mathrm{D}_{3 d}\right)$ in water show to what extent these parameters influence the computation time and the calculated solvation energies $E_{\text {solv }}$.

It can be seen from Table I that $E_{\text {solv }}$ is not very sensitive to the level of tesserae division $N D$. Because the computation time increases with ND, a low value is recommended. Table II shows that the first loop of self-polarization contributes most significantly to $E_{\text {solv }}$. Further increase of $N L$ increases the computation time but does not change substantially $E_{\text {solv }}$.

The computation time is sensitive to the value of $R_{\text {min }}$ (Table III). Thus, increasing $R_{\text {min }}$ from 0.2 to $0.5 \AA$ decreases the total number of spheres and the central processing unit (CPU) time by a factor 8. However, the solvation energy is not so critically dependent on $R_{\min }$. In fact, an increase of the number of spheres smoothes the surface but

TABLE I.

MST-PC Calculations on $\mathrm{PO}(\mathrm{OH})_{3}$ in THF at $\mathrm{NL}=4$, $R_{\min }=0.2 \AA$.

\begin{tabular}{lllll}
\hline$N D$ & $N_{\text {tes }}$ & $S$ & $-E_{\text {solv }}$ & $\begin{array}{c}\text { CPU } \\
\text { time }\end{array}$ \\
\hline 1 & 197 & 112.0 & 31.8 & 4.4 \\
2 & 237 & 106.4 & 32.0 & 6.3 \\
3 & 257 & 106.1 & 32.2 & 7.4 \\
4 & 273 & 105.6 & 32.1 & 8.6 \\
\hline
\end{tabular}

Number of tesseras $\left(N_{\text {tes }}\right)$, molecular surface $\left(\mathbf{S}, \AA^{2}\right)$, solvation energy $\left(E_{\text {solv }}, \mathrm{kcal} / \mathrm{mol}\right), \mathrm{CPU}$ time $^{\ddagger}$ (s) versus the number of tessera's divisions (ND). Calculations performed for CPU time on an EC-1066 computer (Russian).
TABLE II. MST-PC Calculations on $\mathrm{PO}(\mathrm{OH})_{3}$ in THF at $\mathrm{NL}=4$, $R_{\min }=0.2 \AA$.

\begin{tabular}{lcc}
\hline$N L$ & $-E_{\text {solv }}$ & CPU time \\
\hline 0 & 25.0 & 1.4 \\
1 & 30.4 & 3.2 \\
2 & 31.7 & 5.2 \\
3 & 32.0 & 7.2 \\
4 & 32.1 & 3.8 \\
\hline
\end{tabular}

Solvation energy $\left(E_{\text {solv }}, \mathrm{kcal} / \mathrm{mol}\right), \mathrm{CPU}$ time $(\mathrm{s})$ versus the number of loops of self-polarization (NL). EC-1066 computer (Russian) used for calculations.

may cause inconsistency in the self-polarization procedure. $^{31}$

Taking into account the aforementioned results, we used the parameters $N D=1, N L=1$, and $R_{\min }=0.5 \AA$ in the MST-PC calculations of $I-$ VI.

Solvation Energies of Rigid Solutes. To examine the reliability of the MST-PC approach in studying conformational equilibria in solution, we compared the hydration energies of different conformers of I-IV calculated by MST-PC, MST-QM, GB/SA, and MD with explicit water.

For IV-VI we compared the MST-PC solvation energies in acetonitrile with $E_{\text {solv }}$ obtained from MD simulations in acetonitrile represented explicitly. Results of MD simulations of the 18-crown-6 in acetonitrile, cryptand 2.2.2, and $t$-butylcalix [4]arenetetraamide in water and in acetonitrile were taken from refs. 4, 61-63, and 88 .

Ethylene glycol (I), 1,2-difluoroethane (II), and Fluoroacetic acid (III). The syn, gauche, and anti rotational isomers of $\mathrm{I}$-III correspond to the $\mathrm{C}-\mathrm{C}$ torsional angles $0^{\circ}, 60^{\circ}$, and $180^{\circ}$, respectively. The

TABLE III. MST-PC Calculations on 18-Crown-6 $\left(\mathrm{D}_{3 d}\right)$ in Water at $N D=4, N L=4$.

\begin{tabular}{cccccc}
\hline$R_{\text {min }}$ & $N_{\text {sph }}$ & $N_{\text {tes }}$ & S & $-E_{\text {solv }}$ & $\begin{array}{l}\text { CPU } \\
\text { time }\end{array}$ \\
\hline 0.20 & 990 & 2973 & 356.4 & 20.4 & 1165 \\
0.25 & 606 & 2663 & 356.3 & 20.0 & 858 \\
0.30 & 400 & 2260 & 356.2 & 20.1 & 603 \\
0.35 & 278 & 2189 & 356.1 & 20.1 & 356 \\
0.50 & 102 & 1854 & 355.3 & 20.4 & 152 \\
\hline
\end{tabular}

ESP charges from ref. 84: number of spheres $\left(N_{\text {sph }}\right)$ and tesserae $\left(N_{\text {tes }}\right)$, molecular surface $\left(S, \AA^{2}\right)$, solvation energy ( $E_{\text {solv }}, \mathrm{kcal} / \mathrm{mol}$ ), CPU time (s) versus the parameter $R_{\text {min }}$ (Å). EC-1066 computer used for calculations. 
torsional angles $\mathrm{HO}-\mathrm{CC}$ in I and III were kept at $180^{\circ}$.

The results presented in Figure 1 show that the MST-PC method gives the same trend in hydration energies as the more sophisticated MST-QM calculations, displaying better hydration of syn forms of ethylene glycol and 1,2-difluoroethane and the gauche form of the fluoroacetic acid. These results are also consistent with the GB/SA calculations and with MD simulations on I-III in water with rigid solute (Fig. 1). The only difference is found for II: the syn-form is hydrated better than the gauche-form in MST-PC and MST-QM calculations, whereas MD simulations result in their similar hydration.

18-crown-6 (IV). Four conformers were compared (Fig. 2): the $\mathrm{D}_{3 d}$ (all six oxygens lie almost in the same plane ${ }^{94}$ ), $C_{2}$ (extracted from dibenzo-18crown-6; ref. 95), $C_{1}$ (from the 18-crown-6 $\cdot$ NaNCS complex ${ }^{96}$ ), and $C_{i}$ (free molecule in the solid state $^{97}$ ). In the first three conformers the oxygens are more accessible to the solvent than in the $C_{i}$ conformer. ${ }^{98}$ The results (Table IV) show that the order of MST-PC hydration energies $\left(D_{3 d} \geq C_{2} \approx\right.$ $\mathrm{C}_{1} \gg \mathrm{C}_{i}$ ) is consistent with the data of $\mathrm{MD}$ and MC simulations with explicit solvent.

It has been shown previously that the hydration energy of 18-crown-6 is a function of its conformation and cannot be explained either by its dipole moment or by the surface of heteroatoms accessible to the solvent. ${ }^{98}$ Our calculations support fully this view. Indeed, the $\mathrm{D}_{3 d}$ and $\mathrm{C}_{i}$ conformers both have zero dipoles, whereas the latter is hydrated much more weakly. Although the solvent-accessi- ble surface of the $C_{1}$ form ( $\left.327 \AA^{2}\right)$ is less than that of $C_{i}\left(337 \AA^{2}\right), C_{2}\left(347 \AA^{2}\right)$, or $D_{3 d}\left(354 \AA^{2}\right)$, the $C_{1}$, $\mathrm{D}_{3 d}$, and $\mathrm{C}_{2}$ conformers are similarly hydrated.

In acetonitrile, the MST-PC model does not reproduce the trend of total solvation energies obtained in MD simulations with explicit solvent. However, it qualitatively reproduces the trend in the related electrostatic components (see Table IV).

Cryptand 2.2.2 (V). We compare the same four typical conformers as simulated previously by MD in water ${ }^{61}$ and in acetonitrile ${ }^{88,99}$ (Fig. 3): the "in-in" (II) form with converging nitrogens, the "out-out" (OO) and SS forms with diverging heteroatoms, and the $\mathrm{K}$ conformer with converging heteroatoms and diverging $\mathrm{CH}_{2}$ groups, extracted from $\mathrm{K}^{+}$cryptate.

In water, MST-PC calculations show a slightly better hydration of the $\mathbf{K}$ form compared to the others, whereas according to MD simulations, ${ }^{61}$ the II, OO, and SS forms are solvated much less than the $\mathbf{K}$ conformer (see Table V). In acetonitrile, all conformers are similarly solvated both in the MST-PC calculations and in the MD simulations with explicit solvent. ${ }^{63}$

t-Butyl-Calixl4larenetetraamide (V). As in the previous MD study, ${ }^{4,62}$ three conformers were compared: VI(C) with carbonyl groups converging to the $\mathrm{C}_{4}$ symmetry axis (Fig. 4a), VI(D) with diverging carbonyls (Fig. 4b), and VI(free) with two carbonyls converging and the two others diverging (Fig. 4c). Their solvation energies computed both from MD trajectories ${ }^{4,62}$ and by the continuum model are presented in Table VI.

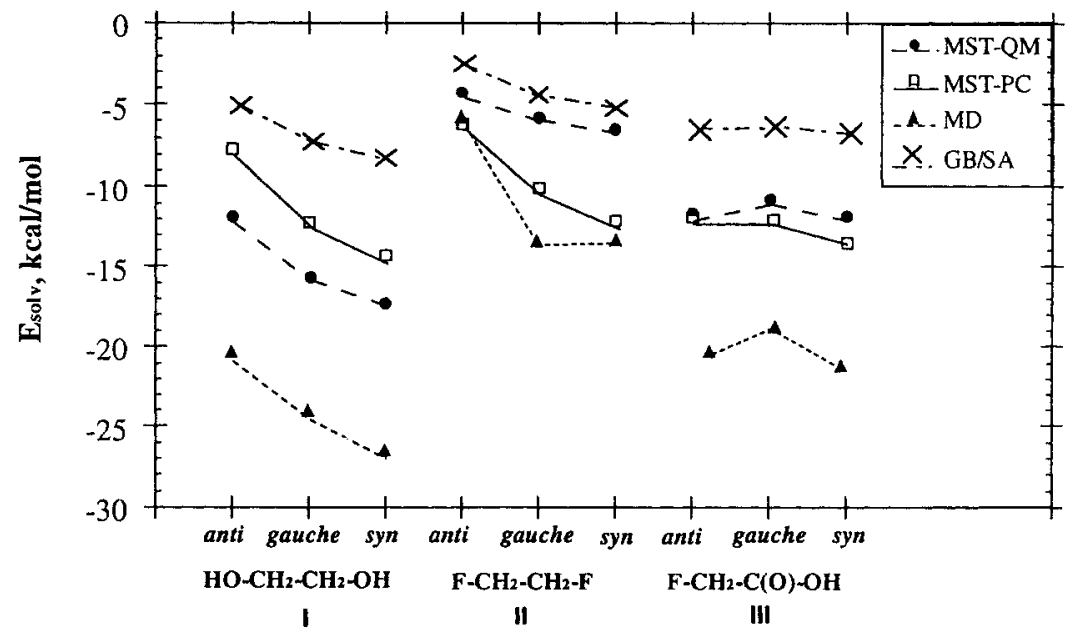

FIGURE 1. Hydration energies of syn, gauche, and trans conformers of ethylene glycol (I), 1,2-difluoroethane (II), and fluoroacetic acid (III) from MST-QM, MST-PC, GB / SA, and MD (AMBER) calculations. 
TABLE IV.

Solvation Energy $\left(-E_{\mathrm{solv}}, \mathrm{kcal} / \mathrm{mol}\right)$, of Four Conformers of 18-Crown-6 in Water and in Acetonitrile.

\begin{tabular}{|c|c|c|c|c|c|}
\hline & & $\mathrm{D}_{3 d}$ & $\mathrm{C}_{2}$ & $\mathrm{C}_{1}$ & $\mathrm{C}_{i}$ \\
\hline \multirow[t]{8}{*}{ In water } & Continuum mode ${ }^{a-c}$ & & & & \\
\hline & $\left({ }^{\mathrm{a}}\right)$ & 19.4 & 19.1 & 17.7 & 8.7 \\
\hline & $\left({ }^{b}\right)$ & 36.9 & 37.3 & 34.0 & 17.7 \\
\hline & $\left({ }^{\mathrm{C}}\right)$ & 13.0 & 14.0 & 12.9 & 5.3 \\
\hline & Explicit water & & & & \\
\hline & $\mathrm{MD}^{\mathrm{d}}$ & $\begin{array}{c}55.8 \\
(34.8)^{\mathrm{h}}\end{array}$ & $\begin{array}{c}55.6 \\
(34.8)\end{array}$ & $\begin{array}{c}53.3 \\
(35.2)\end{array}$ & $\begin{array}{c}35.3 \\
(18.6)\end{array}$ \\
\hline & $M C^{e}$ & 52.4 & & 54.3 & 29.4 \\
\hline & $\mathrm{MC}^{f}$ & 37.1 & & 37.6 & \\
\hline \multirow[t]{3}{*}{ In acetonitrile } & Continuum model $^{a}$ & & & & \\
\hline & $\begin{array}{c}\left({ }^{a}\right) \\
\text { Explicit } \\
\text { acetonitrile }\end{array}$ & 15.7 & 15.9 & 14.9 & 8.7 \\
\hline & $\mathrm{MD}^{\mathrm{g}}$ & $\begin{array}{c}37.2 \\
(12.5)^{h}\end{array}$ & $\begin{array}{l}25.6 \\
(8.1)\end{array}$ & $\begin{array}{c}29.7 \\
(11.4)\end{array}$ & $\begin{array}{l}29.7 \\
(4.6)\end{array}$ \\
\hline
\end{tabular}

In water, $\mathrm{MD}$ simulations result in a clearly larger solvation of the VI(C) form, whereas the continuum model leads to a slight preference of the VI(C) over the VI(D) conformer. The VI(free) form is the worst solvated in both models. In acetonitrile, preferential solvation of the VI(C) form is observed in the MD simulation with explicit solvent, but the VI(D) conformer is better solvated in the continuum model. The trends of MST-PC solvation energies calculated by the MST-PC method are not the same as for electrostatic components of solute--solvent interaction energies in MD simulations.

Geometry Optimization In Vacuo and in Solution. To investigate to what extent the field of the polarized cavity influences the molecular geometry of the solute in the energy minimization procedure, we have compared the optimized structures of ethylene glycol and of macrocycle 18-crown-6 in vacuo and in solution.

MM calculations of ethylene glycol in the gas phase starting from the syn, anti, and gauche forms lead to two minima of 2.0 and $2.5 \mathrm{kcal} / \mathrm{mol}$, which correspond to anti and gauche conformers, respec- tively. Calculations in water result in three conformers with steric energies $5.7 \mathrm{kcal} / \mathrm{mol}$ (syn), 2.5 $\mathrm{kcal} / \mathrm{mol}$ (gauche), and $2.1 \mathrm{kcal} / \mathrm{mol}$ (anti). The torsional OCCO angle of the gauche conformer is $64^{\circ}$ in vacuo and $54^{\circ}$ in water. Taking into account the solvation energy term in addition to the steric energy term changes the order of relative stabilities. The most stable form is gauche $<\operatorname{syn}(\Delta E=$ $4.5 \mathrm{kcal} / \mathrm{mol})<$ anti $(\Delta E=10.8 \mathrm{kcal} / \mathrm{mol})$. This largest stability of the gauche form is consistent with MC simulations of Nagy et al. ${ }^{100,101}$ with explicit water. For the syn form however, the MSTPC solvation energies and stabilities are quantitatively exaggerated, probably because of its largest dipole moment. This is inconsistent with expectations and previous theoretical work. ${ }^{100,101}$ These results clearly show the importance of consistency of the intrinsic energy and solvation energy components of total energy, which probably was not achieved in our calculations.

For macrocycle 18-crown-6, the influence of the continuum solvation on the geometry is small. The diagonal $\mathrm{O} \cdots \mathrm{O}$ distances in $\mathrm{C}_{1}$ and $\mathrm{C}_{i}$ conformers are only $0.1 \AA$ larger in solution than in vacuo, 
<smiles>[111InH]</smiles><smiles>[111In]</smiles>

$D_{3 d}$

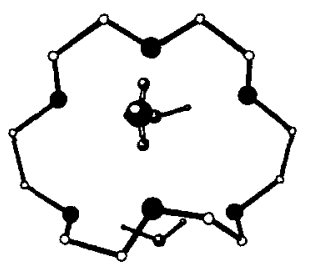

$\mathrm{C}_{1}$

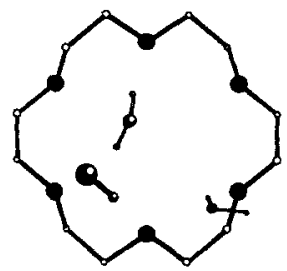

$\mathrm{C}_{2}$
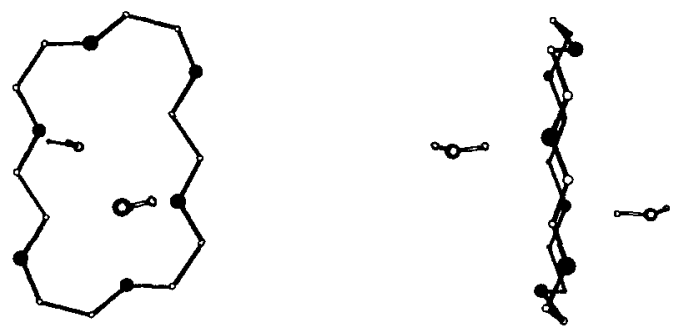

$\mathrm{C}_{\mathrm{i}}$

FGURE 2. $D_{3 d}, C_{1}, C_{2}$, and $C_{i}$ conformers of 18-crown-6 (IV) and their typical hydration pattern (orthogonal views). $\mathrm{CH}$ protons are omitted.

which corresponds to a slightly more open cavity. The optimized geometry of the $\mathrm{D}_{3 d}$ and $\mathrm{C}_{2}$ conformers are nearly the same in vacuo as in solution.

Scope and Limitations of the MST-PC Model. Conformational analysis involves the energy comparison of several conformers of a given molecule in solution, and our results (Tables IV-VI, Fig. 5) show that the MST-PC model reliably reproduces the trends in hydration energies $\left(E_{\mathrm{hydr}}\right)$ of IV-VI,
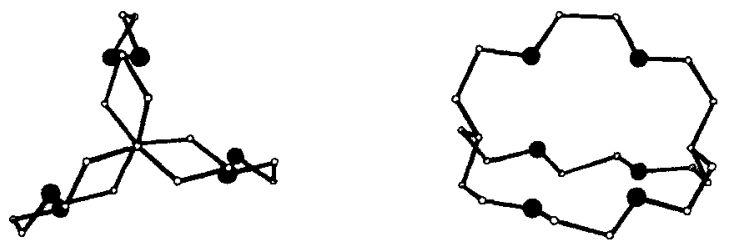

K
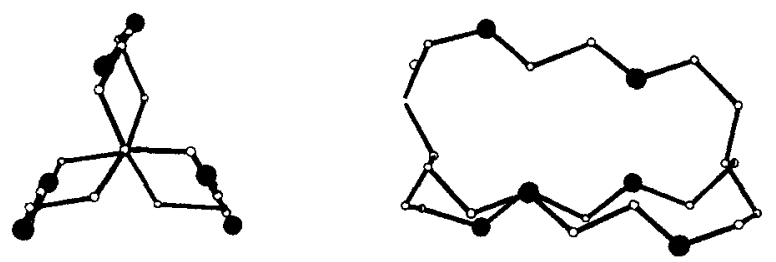

11
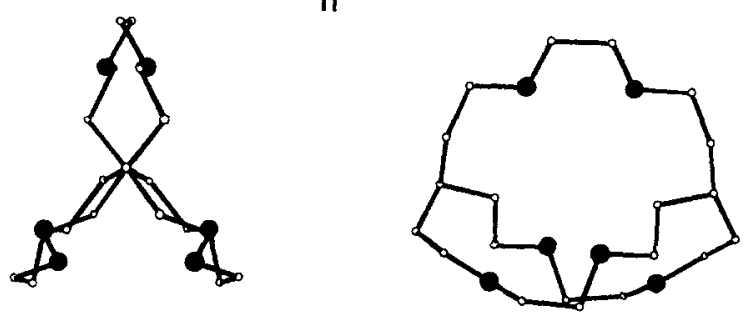

OO
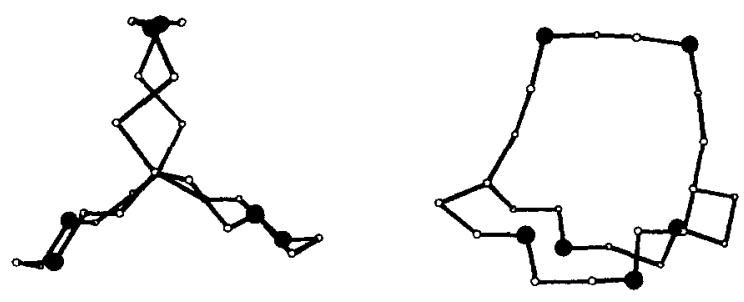

SS

FIGURE 3. K, II, OO, and SS conformers of the 2.2.2 cryptand (V) (orthogonal views). $\mathrm{CH}$ protons are omitted.

compared to MD simulations with explicit solvent. For a given solute, these trends are qualitatively reproduced with different sets of charges and parameters of $N L, N D$, and $R_{\min }$. Thus, calculations on IV with two different sets of charges result in the same order of $E_{\text {hydr }}$ (Table IV). Hydration energies of IV and $\mathbf{V}$ calculated with $N L=3$, $N D=3$, and $R_{\min }=0.2 \AA$ differ by $2-4 \mathrm{kcal} / \mathrm{mol}$ from those calculated ith $N L=1, N D=1$, and $R_{\text {min }}=0.5 \AA$ but display the same trends from one conformer to the other.

To compare solvation energies of different solutes, calculations have to be performed with the 
TABLE V. Solvation Energy $\left(-E_{\text {solv }}, \mathrm{kcal} / \mathrm{mol}\right)$ of Four Conformers of Cryptand 2.2.2 in Water and in Acetonitrile.

\begin{tabular}{|c|c|c|c|c|c|}
\hline & & $\mathrm{K}$ & II & 00 & SS \\
\hline \multirow[t]{5}{*}{ In water } & $\begin{array}{l}\text { Continuum } \\
\text { model }\end{array}$ & & & & \\
\hline & (a) & 16.7 & 14.0 & 12.2 & 12.8 \\
\hline & $\left.{ }^{b}\right)$ & 20.7 & 10.0 & 12.5 & 12.4 \\
\hline & Explicit water & & & & \\
\hline & MD ( $\left.{ }^{C}\right)$ & 79 & 49 & 48 & 42 \\
\hline \multirow[t]{4}{*}{ In acetonitrile } & $\begin{array}{l}\text { Continuum } \\
\text { model }\end{array}$ & & & & \\
\hline & $\left({ }^{a}\right)$ & 12.9 & 12.9 & 11.3 & 12.4 \\
\hline & $\begin{array}{c}\text { explicit } \\
\text { acetonitrile }\end{array}$ & & & & \\
\hline & $\mathrm{MD}\left({ }^{d}\right)$ & $\begin{array}{c}74.1 \\
(13.9)^{\mathrm{e}}\end{array}$ & $\begin{array}{l}67.1 \\
(9.6)\end{array}$ & $\begin{array}{l}68.5 \\
(8.7)\end{array}$ & $\begin{array}{l}69.9 \\
(7.6)\end{array}$ \\
\hline
\end{tabular}

same parameters $N L, N D$, and $R_{\min }$ and with consistent sets of charges. In fact, the MST-PC calculations performed on IV-VI meet these requirements because the ESP charges were computed consistently (in 6-31G* ab initio calculations for IV ${ }^{84}$ and $V_{r}^{61}$ and by the MNDO method for VI $^{62}$ with further scaling to $a b$ initio charges according to ref. 102). From the data plotted in Figures 5 and 6, one can see that the MST-PC model is successful in reproducing qualitatively trends in the hydration energies from one molecule to the others, compared to MD simulations with explicit solvent. For instance, calixarene VI is better hydrated than crown ether IV and cryptand V. With explicit, as well as with continuum models, the II, OO, and SS forms of $\mathbf{V}$ are better hydrated than the $\mathrm{C}_{i}$ conformer of IV but worse than the $\mathrm{D}_{3 d}, \mathrm{C}_{1}$, and $\mathrm{C}_{2}$ conformers of 18-crown-6. The only exception concerns the $\mathbf{K}$ form of cryptand, which is hydrated better than IV in the simulations with explicit water, whereas in MST-PC calculations its hydration energy is less negative than that for the $\mathrm{D}_{3 d}$ conformer of IV (Fig. 5).

The GB/SA continuum model also reproduces the trends in hydration energies of IV-VI obtained in MD simulations with explicit water without exception (see Fig. 5). The changes in the GB/SA solvation energies $E_{\text {solv }}$ from one molecule to the other are due mostly to the electrostatic term; the cavitation/van der Waals components of $E_{\text {solv }}$ remained similar for a given solute. The success of this simple approach is surprising given the neglect of self-polarization at the surface of the solute. On the other hand, including such a selfpolarization effect in the more sophisticated MSTPC method may lead to artefacts if the solute has clefts and pockets or if the molecular surface contains concave fragments. This may cause, in particular, poor convergence of the iterative self-polarization procedure ${ }^{103}$ and hence lead to biased charges on the tesserae and to incorrect $E_{\text {solv }}$. In fact, the surfaces of the VI(C) conformer of calixarene and of the $K$ form of cryptand $\mathbf{V}(\mathbf{K})$ display such concavities (Fig. 7). It is clear from these considerations that the MST-PC method may meet problems in relation to the particular topology of the solute.

Another limitation of the MST approach is that it restricts solvent-solute interactions to the electrostatic component and neglects the van der Waals contribution. This could explain why it works reasonably well for aqueous solutions but not for acetonitrile (Fig. 6). Indeed, MD simulations of crown ether IV and calixarene VI in solution show that the electrostatic term contributions 65 to $80 \%$ to the solvent-solute interaction energy in water ${ }^{62}$ but only 15 to $35 \%$ in acetonitrile ${ }^{4,63}$ (see Tables IV and VI). Therefore, in acetonitrile the MST-PC model reproduces the trend in electrostatic solvation energies for 18-crown-6 rather than in total solvent-solute interaction energies (Fig. 6). As 

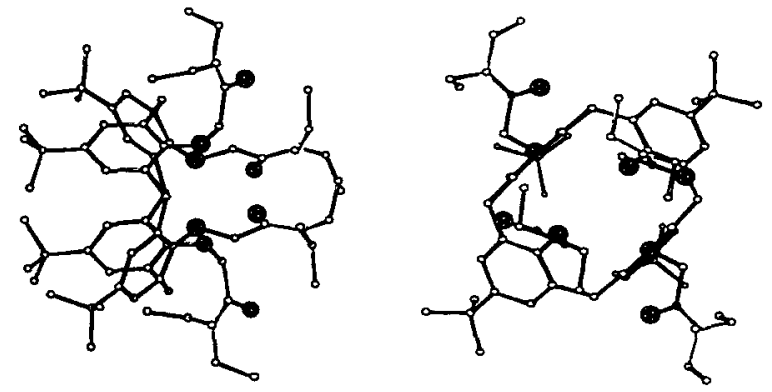

$\mathrm{V} \mid(C)$
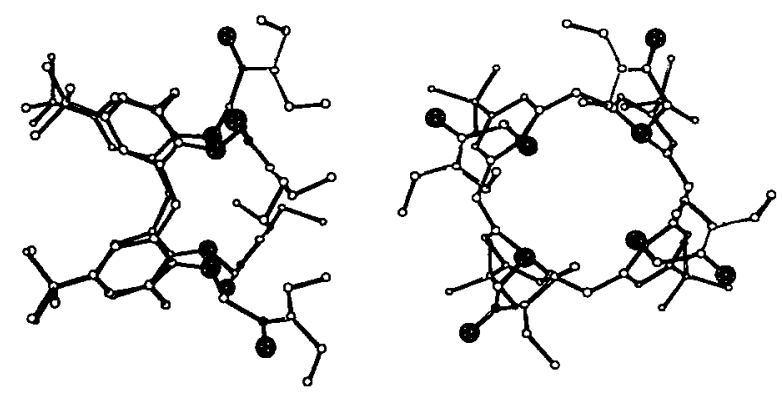

VI(D)

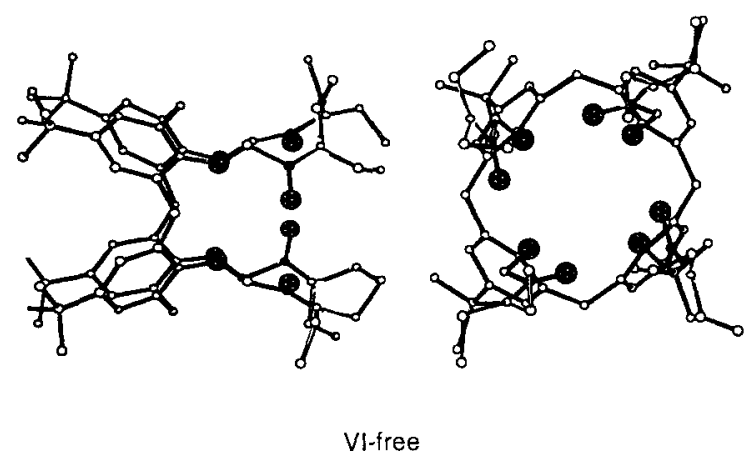

FIGURE 4. VI(C), VI(D), and VI(free) conformers of $t$-butyl-calix[4]arene tetramide (orthogonal views). $\mathrm{CH}$ protons are omitted.

noted above for calixarene VI in acetonitrile, the MST-PC relative solvation energies do not follow the electrostatic component of the solute-solvent interaction energies obtained from explicit MD simulations. This may be due to specific solvation patterns. In addition, the incorporation of van der Waals components in the solvation energy becomes a clear necessity. In fact, the algorithm developed by Floris and Tomasi ${ }^{104}$ includes this component and allows one for the energy minimization. Recently Oronzco, Jorgensen, and Luque $^{105}$ have shown that the MST-QM model combined with a van der Waals term gives good values of solvation energies. The cavitation energy, which qualitatively corresponds to the relaxation energy of the solvent, ${ }^{40}$ may be also included in the force field. ${ }^{14,31,58,106}$

\section{Discrete / Continuum Representation of the Solvent}

Importance of the Explicit First-Shell Solvation. Specific solvation may play an important role in stabilization of particular conformers of a solute in solution. ${ }^{63,88}$ Thus, the early Monte Carlo simulation on the 18-crown- $6+100 \mathrm{H}_{2} \mathrm{O}$ cluster $^{98}$ gave a clear demonstration of a specific hydration pattern of the crown as a function of its conformation. The importance of bridging hydrogen-bonded pattern for some conformers $\left(D_{3 d}\right.$ and $\left.C_{1}\right)$ was shown. These results are confirmed in the MD simulations. ${ }^{107}$ Specifically, two water molecules bridge two pairs of oxygens for the $\mathrm{D}_{3 d}$ and $\mathrm{C}_{2}$ forms, and there are three such bridges for the $C_{1}$ form. The $C_{i}$ form does not allow the bridging, but only for a few linear hydrogen bonds. Analogously, three bridging water molecules have been characterized in the $\mathbf{K}$ form of the 2.2.2 cryptand, leading to better hydration of this conformer compared to others. $^{61}$ In the MD simulations of the VI(C) form of $t$-butyl-calix[4]arenetetraamide in water (Fig. 8), one solvent molecule diffuses into the hydrophilic pseudocavity at lower rim and bridges the phenolic oxygens. In this conformer, four axially oriented amidic fragments form a hydrophilic pocket, which is filled by seven or eight water molecules. Favorable interaction of these solvent molecules with the solute results in better solvation of the VI(C) form compared to the VI(D) and VI(free) conformers, in which there is no hydrophilic pocket.

In fact, specific solute-solvent interactions are present in nonaqueous solvents as well. For instance, in the MD simulations of calixrene VI in acetonitrile, one $\mathrm{MeCN}$ molecule is found inside the cone of the three conformers. In the VI(C) form, a second $\mathrm{MeCN}$ molecule is found in the pseudo cavity at the lower rim, pointing its methyl group toward the phenolic oxygens. ${ }^{4}$ Such solvent molecules may be considered as part of the solute.

The preceding examples show that the continum models meet problems when the solute binds firmly some solvent molecules (either at specific groups or via multiple cooperative interactions). In such cases, solvation models which consider the first solvation shell of the solute explicitly and the remaining solvent as a polarized continuum appear, therefore, more realistic than pure continuum methods. Another advantage of such 
TABLE VI.

Solvation Energy $\left(-E_{\mathrm{solv}}, \mathrm{kcal} / \mathrm{mol}\right)$ of Three Conformers of $\mathrm{t}$-butyl-calix[4]arenetetraamide in Water and in Acetonitrile.

\begin{tabular}{|c|c|c|c|c|}
\hline & & $\mathbf{V I}(\mathbf{C})$ & VI(D) & VI(free) \\
\hline \multirow[t]{5}{*}{ In water } & Continuum model $\left.\right|^{a, b}$ & & & \\
\hline & (a) & 51.0 & 48.7 & 38.4 \\
\hline & (b) & 66.0 & 27.3 & 26.7 \\
\hline & Explicit water & & & \\
\hline & $\operatorname{MD}\left({ }^{c}\right)$ & $\begin{array}{c}188.3 \\
(127.2)^{\mathrm{e}}\end{array}$ & $\begin{array}{l}140.1 \\
(89.9)\end{array}$ & $\begin{array}{l}133.7 \\
(78.6)\end{array}$ \\
\hline \multirow[t]{3}{*}{ In acetonitrile } & Continuum model & & & \\
\hline & $\begin{array}{c}\left({ }^{a}\right) \\
\text { Explicit } \\
\text { acetonitrile }\end{array}$ & 40.8 & 44.5 & 39.1 \\
\hline & $M D\left({ }^{d}\right)$ & $\begin{array}{c}108.5 \\
(33.0)^{\mathrm{e}}\end{array}$ & $\begin{array}{c}99.3 \\
(24.6)\end{array}$ & $\begin{array}{c}98.1 \\
(21.6)\end{array}$ \\
\hline
\end{tabular}

${ }^{\mathrm{a}, \mathrm{b}}$ Calculations of solvation energies with continuum models: MST-PC (ESP charges from ref. $62 ; R_{\min }=0.5 \AA ; N D=1, N L=1$, superscript a), and GB / SA (superscript b).

${ }_{c, d}$ MD simulations with AMBER, 50 ps; data rom refs. 62 (superscript c) and 4 (superscript d)

${ }^{\theta}$ Electrostatic contribution.
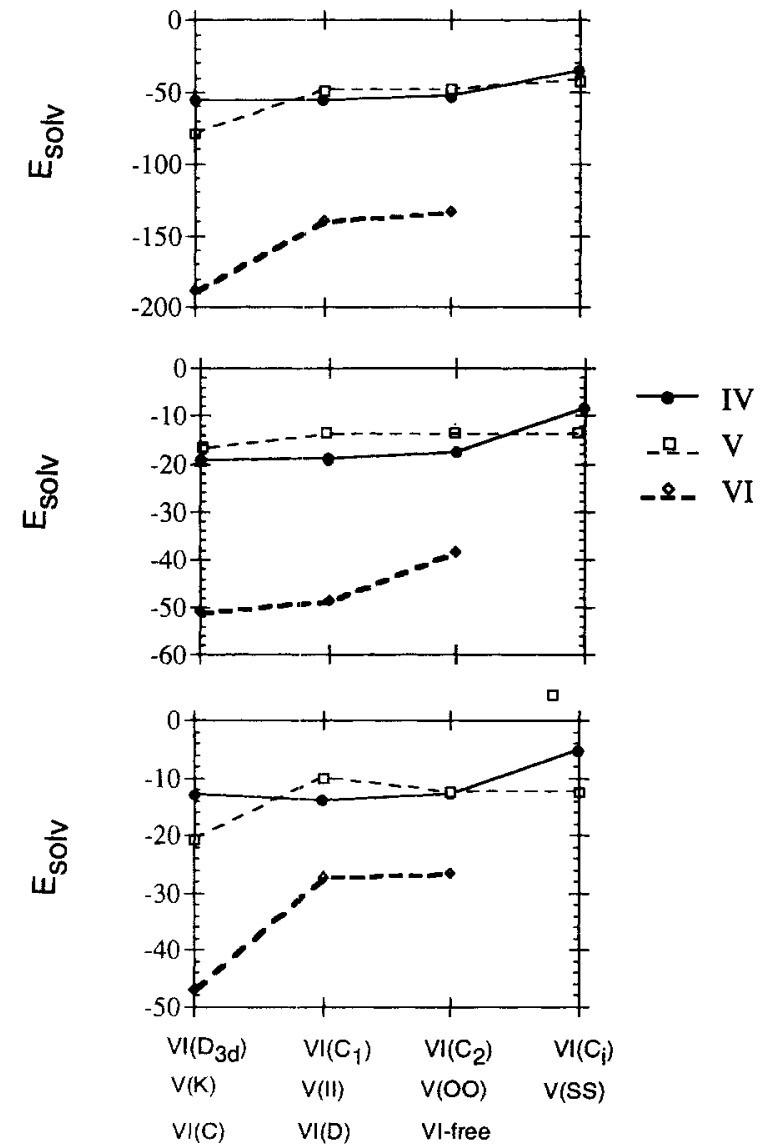

FIGURE 5. Hydration energies of four conformers of IV, four conformers of $\mathbf{V}$, and three conformers of $\mathbf{V} \mathbf{I}$ calculated with AMBER (top), MST-PS (middle), and GB / SA (bottom). hybrid discrete/continuum methods is that when some solvent molecules fill pockets and clefts of the solute, the surface of this supermolecule becomes smoother.

Choice of the First Solvation Shell. A critical feature of the discrete/continuum calculations concerns the modeling of the first solvation shell. It was suggested ${ }^{108-110}$ that the positions of solvent molecules coincide with local minima on the hypersurface of solvent-solute interaction energy. Although this approach was successfully used in the study of hydration of uracile and cytosine, ${ }^{109,110}$ its validity is limited because it does not take into account interactions between solvent molecules and the flexibility of the solute. For polydentate solutes, the number of energy minima depends on the conformational state and may not correspond to the number of solvent molecules in the solvation shell. For instance, only two minima were observed for the interaction of one water molecule with 18-crown-6 $\left(D_{3 d}\right),{ }^{98}$ whereas according to Monte Carlo ${ }^{98}$ or MD simulations there are four water molecules in the first shell of ether oxygens.

In our discrete/continuum calculations of the 18-crown-6 and calixrene VI, the starting structure of solute + first solvation shell was taken from MD simulations in a box of water molecules. Water molecules $\left(n=4\right.$ for the $\mathrm{D}_{3 d}$ form, $n=3$ for the $C_{1}$ and $C_{2}$ forms, $n=2$ for the $C_{i}$ form; see Table VII and Fig. 2) which form hydrogen bonds with ether oxygens were selected from the last 

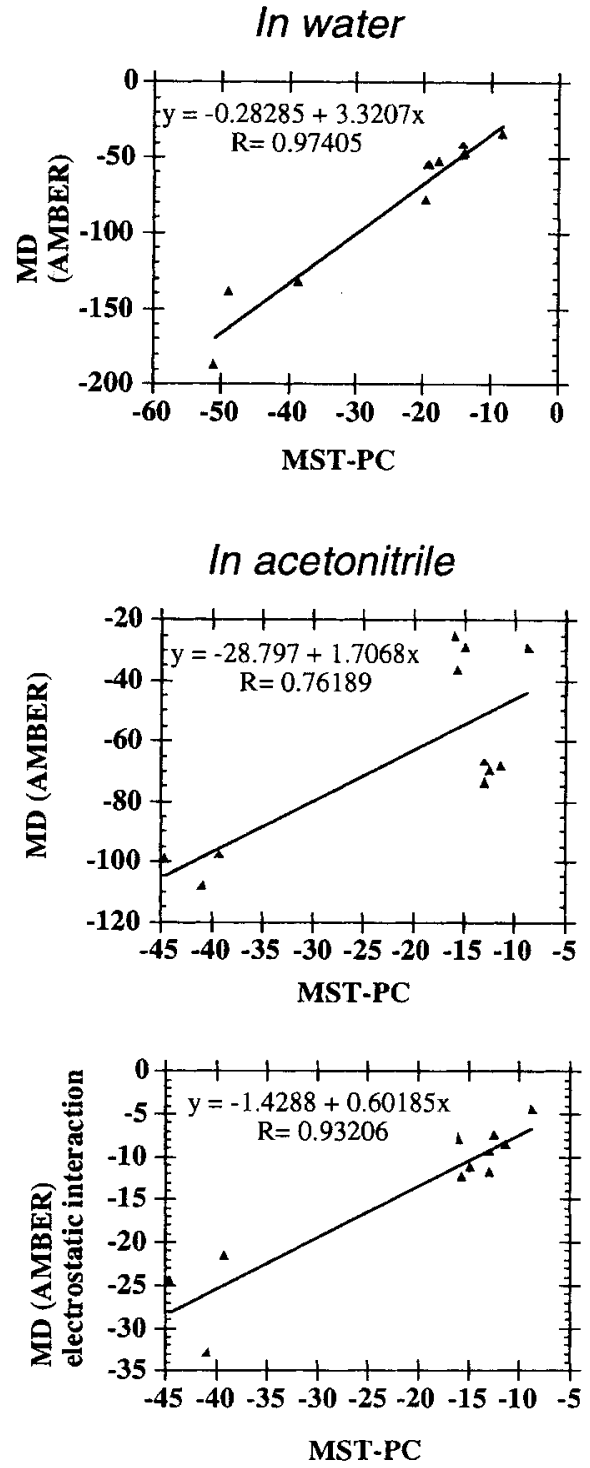

FIGURE 6. Correlation between solvation energies of IV-VI in water (top) and acetonitrile (middle and bottom) obtained in MST-PC calculations and in MD simulations (AMBER) with explicit solvent (values in $\mathrm{kcal} / \mathrm{mol}$ ). In the bottom figure, only the electrostatic component of the solute--solvent interaction energies (AMBER) is considered.

coordinate sets of the 50-ps MD simulations. After energy minimization with AMBER, the clusters solute $+n \mathrm{H}_{2} \mathrm{O}$ were used in MM calculations with MM2IS.

The clusters $\mathrm{VI}+8 \mathrm{H}_{2} \mathrm{O}$ (with two water molecules per carbonyl group) were selected from the last coordinate sets of the 100-ps MD simulations of the VI(C) and VI(D) forms of $t$-butylcalix[4]arenetetraamide in explicit water. The hydration patterns of these conformers are very dif- ferent. For VI(D), each carbonyl group is hydrogen bonded to two water molecules. For VI(C), all water molecules are situated inside the pseudocavity formed by four converging amidic groups.

Consistency of the Force Field Solvation Terms. In the discrete/continuum calculations, it is desirable to achieve consistency of the discrete $\left(E_{\mathrm{discr}}\right)$ and continuum $\left(E_{\text {cont }}\right)$ components of solvation energy in relation to the explicit all-solvent representation. $E_{\text {discr }}$ and $E_{\text {cont }}$ should correspond, respectively, to the contribution of the first solvation shell [ $E(1 \mathrm{st}$ shell)] and of the remaining solvent molecules [ $E$ (outer)]. This can be achieved by writing

$$
E_{\mathrm{solv}}=E_{\mathrm{discr}}+K_{E} \cdot E_{\mathrm{cont}}
$$

where the scaling coefficient $K_{E}$ is to put discrete and continuum energies on the same scale. We calibrated $K_{E}$ assuming that the relative contribution of the first solvation shell should be the same in AMBER (explicit all-solvent MD calculations) as in MM2IS calculations (MST-PC solvation model).

$$
\begin{aligned}
& \frac{E(1 \text { st shell })}{E(1 \text { st shell })+E \text { (outer })}(\text { AMBER }) \\
& =\frac{E_{\text {discr }}}{E_{\text {discr }}+K_{E} \cdot E_{\text {cont }}} \text { (MM2IS) }
\end{aligned}
$$

Combining AMBER and MM2IS calculations on four clusters of 18-crown- $6\left(D_{3 d}, C_{1}, C_{2}\right.$, or $C_{i}$ conformers) $+n \mathrm{H}_{2} \mathrm{O}$ led to an average value of $K_{E}=2.8$.

The solvation energies calculated by eq. (10) using both $K_{E}=1$ (unscaled energy $E_{\text {solv }, 1}$ ) and $K_{E}=2.8$ (scaled energy $E_{\text {solv }, 2}$ ) are presented in Table VII. One can see that the major difference in hydration energies of 18-crown-6 as a function of its conformation comes from its interactions with the first hydration shell. The outer solvation effects are similar for all forms.

To examine the consistency of the model, we performed with MM2IS several calculations of $E_{\text {solv }}$ using eq. (10) on clusters containing the same solute and different numbers of water molecules $n$. If consistency is reached, the solvation energy should not depend on $n$ because the decrease (increase) of the discrete component of $E_{\text {solv }}$ is compensated by the increase (decrease) of the continuum one. Data presented in Table VII show that scaled solvation energies $E_{\mathrm{solv}, 2}$ of 18-crown-6 are similar for the clusters containing two to four or six water molecules. On the other hand, unscaled solvation energies $E_{\mathrm{solv}, 1}$ are different for these clusters. 

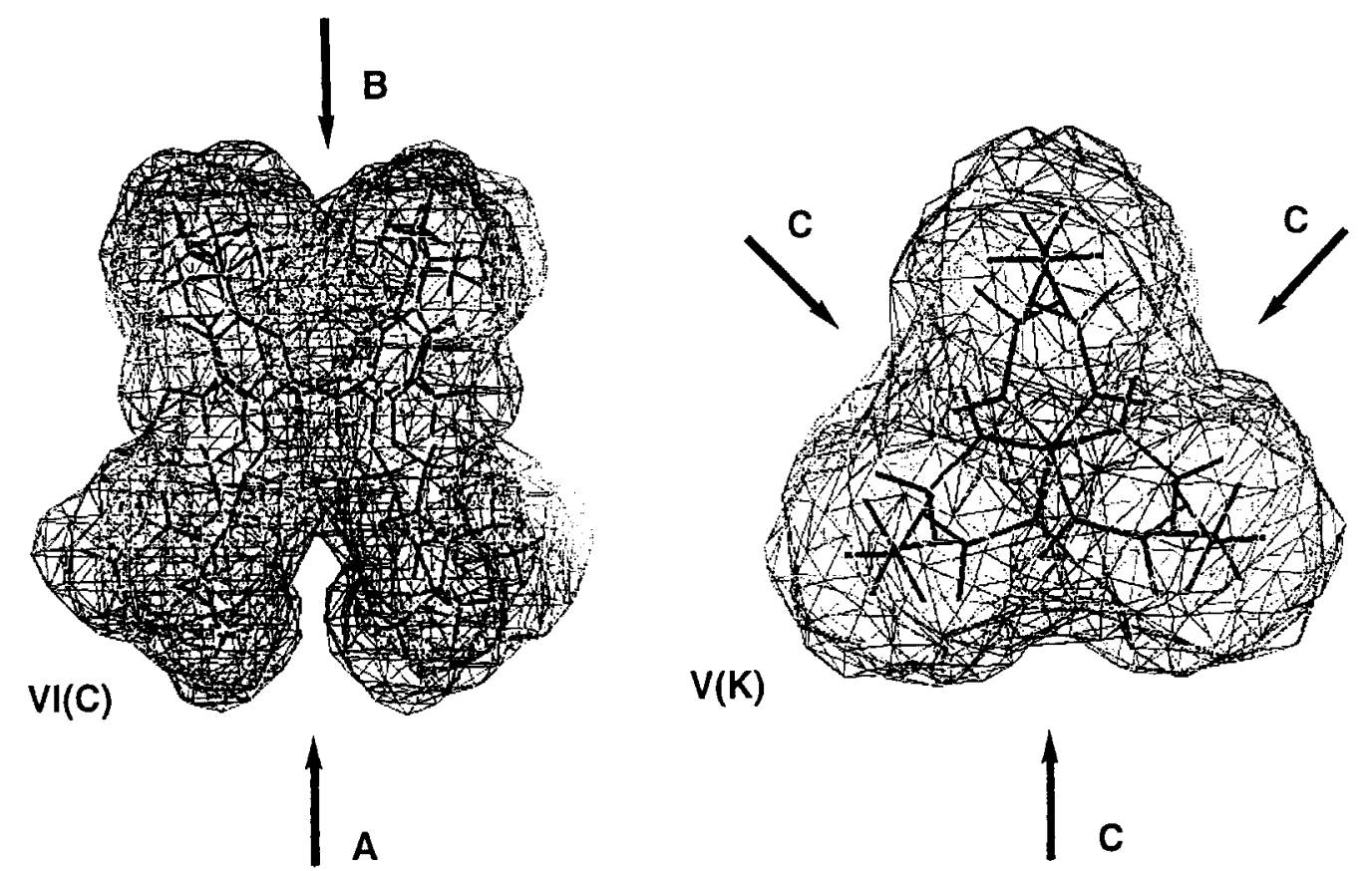

FIGURE 7. Molecular surfaces of the VI(C) conformer of $t$-butyl-calix[4]arenetetraamide (left) and the $\mathrm{K}$ conformer $\mathbf{V}(\mathbf{K})$ of the 2.2.2 cryptand (right). Arrows show the pockets (A and $\mathbf{B})$ in $\mathbf{V I}(\mathbf{C})$ and concave parts (C) of the molecular surface of $\mathbf{V}(\mathbf{K})$.

Results of the discrete/continuum calculations on $t$-butyl-calix[4]arenetetraamide including eight first-shell water molecules (Fig. 8) are presented in Table VIII. One can see that the VI(C) form is now much better hydrated than VI(D), as found in the
MD simulations with explicit solvent. This represents, therefore, a significant improvement relative to purely continuum calculations, in which only a slight preference of $\mathrm{VI}(\mathrm{C})$ over $\mathrm{VI}(\mathrm{D})$ was observed.

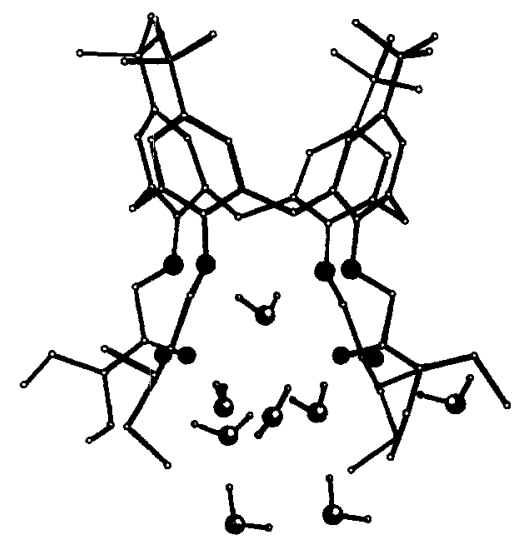

$\mathrm{VI}(\mathrm{C})$

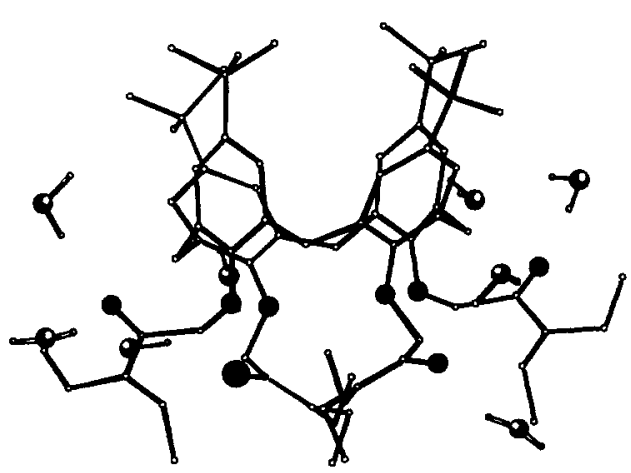

$\mathrm{VI}(\mathrm{D})$

FIGURE 8. $\mathrm{VI}(\mathrm{C})$ and $\mathrm{VI}(\mathrm{D})$ conformers of $t$-butyl-calix[4]arenetetraamide with their first hydration shells modeled by eight water molecules. 
TABLE VII.

Hydration Energies (kcal/mol) of 18-Crown-6 (IV) Conformers in Discrete/Continuum Model.

\begin{tabular}{|c|c|c|c|c|c|c|c|c|c|c|c|c|}
\hline & & $\mathrm{MB}$ & ER (IV in wate & box) & & IST-PC ( & $\mathrm{V}+\mathrm{nH}_{2} \mathrm{C}$ & & & MST-PC & $\left(\mathrm{IV}+6 \mathrm{H}_{2}\right.$ & ) \\
\hline S & 55.9 & 4 & 29.5 & 26.4 & 29.4 & 92 & 38.6 & 556 & 35.4 & 6.6 & 420 & 542 \\
\hline$C_{1}$ & 55.4 & 3 & 27.7 & $2 \varepsilon$ & 24.6 & 10.1 & 34.7 & 53.4 & 31.3 & 8.0 & 39.3 & 54.1 \\
\hline $\mathrm{C}_{2}$ & 56.1 & 3 & 27.0 & 29.1 & 27.4 & 10 & 37.4 & 55.9 & 33.2 & 7.5 & 40.7 & 54.2 \\
\hline $\mathrm{C}_{i}$ & 37.9 & 2 & 8.3 & 29.6 & 7.2 & 8.1 & 15.3 & 30.2 & 9.4 & 8.3 & 17.7 & 33.1 \\
\hline
\end{tabular}

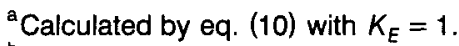

${ }^{b}$ Calculated by eq. (10) with $K_{E}=2.8$.

${ }^{c} n=4$ (for $D_{3 d}$ form), 3 (for $C_{1}$ and $C_{2}$ forms), and 2 (for $C_{i}$ form).

TABLE VIII.

Hydration Energies (kcal / mol) of Two Conformers of $t$-butyl-calix[4]arenetetraamide (VI) in the Discrete /Continuum Model.

\begin{tabular}{lccccc}
\hline & $-E_{\text {discr }}$ & $-E_{\text {cont }}$ & $-E_{\text {solv, }{ }^{\mathrm{a}}}$ & $-E_{\text {solv, }{ }^{\mathrm{b}}}$ & $-E_{\text {solv }}{ }^{\mathrm{c}}$ \\
\hline $\mathrm{VI}(\mathrm{C})$ & 64.2 & 36.9 & 101.1 & 167.8 & 188.3 \\
$\mathrm{VI}(\mathrm{D})$ & 36.6 & 20.5 & 57.1 & 94.0 & 140.1 \\
\hline
\end{tabular}

${ }^{a}$ Calculated by eq. (10) with $K_{E}=1$.

${ }^{b}$ Calculated by eq. (10) with $K_{E}=2.8$.

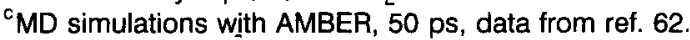

\section{MOLECULAR DYNAMICS WITH THE MST-PC MODEL IMPLEMENTED}

In the MD simulations with continuum solvent, we mostly focus on the aspects of equilibration of the system and reduction of computation time. The MD simulation of urea in water was performed for 80 ps with the parameters $N L=3$, $N D=2$, and $R_{\min }=0.5 \AA$. No drift in total energy $\left(E_{\text {tot }}\right)$ was observed after 8-10 ps. The solvent-solute interaction energy becomes equilibrated in 2 ps (Fig. 9).

The computation time increases dramatically

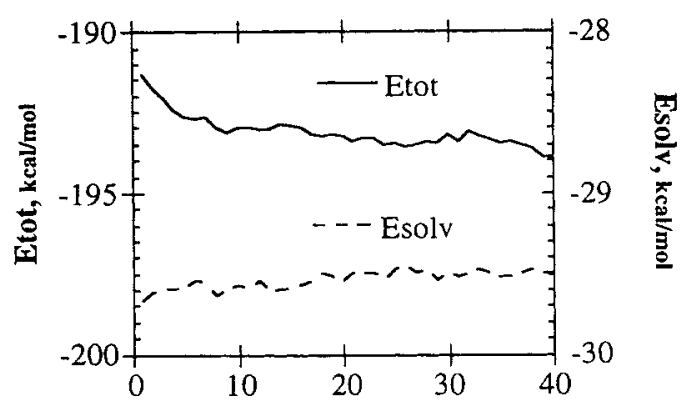

Simulation time, ps

FIGURE 9. Molecular dynamics of urea in water in the continuum model: the total energy and solvation energy versus the simulation time. with the number of atoms of the solute. One timestep of MD with the same parameters $(N L=3$, $N D=2$, and $R_{\min }=0.5 \AA$ ) takes $2.8 \mathrm{~s}$ for urea and $77.3 \mathrm{~s}$ for 18-crown-6 $\left(\mathrm{C}_{i}\right){ }^{\dagger}$ For 18-crown-6 $\left(\mathrm{C}_{i}\right)$, determining the cavity's shape takes $15 \%$ of the total computation time, the forces and energies take about $2 \%$, and the self-polarization procedure takes $83 \%$. The computation time of the selfpolarization procedure [see eqs. (1)-(3)] is proportional to $N_{\text {tes }}^{2}$, where $N_{\text {tes }}$ is the number of tesseras. $N_{\text {tes }}$ grows faster than the number of atoms. For instance, for 18-crown-6 (42 atoms), the program adds 220 spheres and 1445 tesserae, whereas for urea there are only the 8 original spheres represented by 188 tesserae, without any additional spheres.

To reduce the computation time, some calculations were performed for 18-crown-6 with $N D=1$ and $N L=1$. In that case, a timestep takes "only" $12 \mathrm{~s}$, which is still three times larger than that in a standard MD run on 18-crown-6 in a $256 \mathrm{H}_{2} \mathrm{O}$ box. ${ }^{111}$

A possible way to reduce the computation time would be to update the geometry of the cavity every $X$ timesteps only while calculating charges on the tesserae at each timestep. We simulated

\footnotetext{
${ }^{\dagger}$ Calculations have been performed using a Silicon Graphics 4D-320 workstation.
} 
urea in water in five MD simulations of 20 ps with $X=1$ to 5 . The results showed that the cavity's surface and the solvation energy are similar in the five runs, but the computation time is not significantly reduced: from $0.715 \mathrm{~s} /$ timestep $(X=1)$ to $0.610 \mathrm{~s} /$ timestep $(X=5)$.

We have not studied which value of $X$ might give the best compromise between CPU time and accuracy. However, further increase of the value $X$ can be inappropriate because forces arising from the tesserae might be inconsistent with the molecular geometry. This must lead to large fluctuations of the kinetic energy. We observed such fluctuations in simulations with $X=5$, and we updated simultaneously the charges on the tesserae and the cavity shape.

\section{Conclusions}

The molecular mechanics calculations incorporating the MST-PC model have been performed on a set of molecules in different conformations and compared with the average solute-solvent interaction energies from MD simulations with explicit representation of the solvent. Comparison was also performed with the empirical approach used in the MacroModel software. We found that the pure continuum model of the solvent gives generally reliable trends of solvation energies and allows one to study conformational equilibria in water with low computational cost. Some discrepancies have been noticed, however, when the shape and topology of the solute allowed some first-shell water molecules to be firmly bound and to behave as a part of the solute. This led us to investigate the discrete/continuum approach, in which the first solvation shell is added explicitly to the solute. This provides more realistic results, as shown for 18-crown-6 and $t$-butyl-calix[4]arenetetraamide in water. To reach a consistency between the discrete and continuum energy terms, we suggested that one should scale the energy of outer continuum solvation.

Because in the MST approach only the electrostatic component of the solvent-solute interaction energy is calculated, this approach works reasonably well for aqueous solutions but fails for acetonitrile, in which the contribution of van der Waals interactions is higher relative to $E_{\text {solv }}$. In such nonaqueous solvents, the van der Waals solute-solvent interactions must be accounted for explicitly.
The MST-PC method has been implemented in the MM2IS program package, which is designed to perform molecular mechanics calculations of rather complex systems (molecules, aggregates, etc.) in solution using continuum or discrete/continuum representations of the solvent with neutral or charged solutes.

Preliminary MD simulations on urea and 18crown-6 in water have been performed within the MST-PC continuum model of the solvent, incorporated in the GROMOS software. Further investigations are needed to make this solvation model more efficient.

\section{Acknowledgments}

G. W. and A. V. thank CNRS and IDRIS for allocating computer time, Prof. G. Morosi (University of Milan), Drs. J. Alagona and C. Ghio (University of Pisa) for their help with MST-QM calculations, and Dr. T. Simonson for linguistic assistance. Prof. J. Tomasi is acknowledged for making the MGPISA software available to us and for fruitful discussions. A. V. is grateful to NATO for a research grant.

\section{References}

1. S. C. Harvey, Proteins: Structur., Functions, Gen., 5, 78 (1989).

2. A. Pullman and B. Pullman, Q. Rev. Biophys., 7, 505 (1975).

3. J. Lautz, H. Kessler, W. F. van Gunsteren, H.-P. Weber, and R. M. Wenger, Biopolymers, 29, 1669 (1990).

4. A. A. Varnek and G. Wipff, J. Phys. Chem., 97, 10840 (1993).

5. Y. L. Ha and A. K. Chakraborty, I. Phys. Chem., 95, 10781 (1991).

6. H. Sklenar, F. Eisenhaber, M. Poncin, and R. Lavery, In Theoretical Biochemistry \& Molecular Biophysics, D. L. Beveridge and R. Lavery, Eds., Adenine Press, Guilderland, NY, 1990, p. 317.

7. F. S. Lee, Z. T. Chu, and A. Warshel, J. Comp. Chem., 14, 161 (1993).

8. I. Tvaroska, A. Imberty, and S. Pérez, Biopolymers, 30, 369 (1990).

9. M. E. Davis and J. A. McCammon, Chem. Rev., 90, 509 (1990).

10. M.-J. Huron and P. Claverie, J. Phys. Chem., 78, 1862 (1974).

11. M.-J. Huron and P. Claverie, J. Phys. Chem., 78, 1853 (1974).

12. M.-J. Huron and P. Claverie, J. Phys. Chem., 76, 2123 (1972).

13. P. Claverie, J. P. Daudey, J. Langlet, B. Pullman, D. Piazzola, and M. J. Huron, J. Phys. Chem., 82, 406 (1978). 
14. J. Langlet, P. Claverie, J. Caillet, and A. Pullman, J. Phys. Chem., 92, 1617 (1988).

15. D. G. Levitt, Biophys. J., 22, 209 (1978).

16. A. A. Rashin and K. Namboodiri, J. Phys. Chem., 91, 6003 (1987).

17. A. A. Rashin, Int. J. Quant. Chem.: Quant. Biol. Symp., 15, 103 (1988).

18. A. A. Rashin, J. Phys. Chem., 94, 1725 (1990).

19. R. J. Zauhar and R. S. Morgan, J. Mol. Biol., 186, 815 (1985).

20. R. J. Zauhar and R. S. Morgan, J. Comp. Chem., 11, 603 (1990).

21. R. J. Zauhar and R. S. Morgan, J. Comp. Chem., 9, 171 (1988).

22. Y. N. Vorobjev, J. A. Grant, and H. A. Scheraga, J. Am. Chem. Soc., 114, 3189 (1992).

23. M. E. Davis and J. A. McCammon, I. Comp. Chem., 12, 909 (1991).

24. M. E. Davis and J. A. McCammon, J. Comp. Chem., 11, 401 (1990).

25. S. Miertus, E. Scrocco, and J. Tomasi, J. Chem. Phys., 55, 117 (1981).

26. S. Miertus and J. Tomasi, J. Chem. Phys., 65, 239 (1982),

27. R. Cimiraglia, S. Miertus, and J. Tomasi, Chem. Phys. Lett., 80, 286 (1981).

28. F. J. O. del Valle, M. Aguilar, S. Tolosa, J. C. Contador, and J. Tomasi, Chem. Phys., 143, 371 (1990).

29. R. Cammi, F. J. O. del Valle, and J. Tomasi, Chem. Phys., 122, 63 (1988).

30. G. Alagona, R. Bonaccorsi, C. Ghio, R. Montagnani, and J. Tomasi, Pure Appl. Chem., 60, 231 (1988).

31. J. Tomasi, Int. J. Quant. Chem.: Quant. Biol. Symp., 18, 73 (1991).

32. R. Bonaccorsi, P. Palla, and J. Tomasi, J. Am. Chem. Soc., 106, 1945 (1984).

33. J. Tomasi, G. Alagona, R. Bonaccorsi, and C. Ghio, In Molecular Modelling of Structures and Properties of Molecules, Z. Marksic, Ed., Ellis Horwood Ltd., Chichester, 1987, p. 330.

34. G. P. Ford and B. Wang, J. Comp. Chem., 13, 229 (1992).

35. G. P. Ford and B. Wang, J. Am. Chem. Soc., 114, 10563 (1992).

36. F. J. Luque, M. J. Negre, and M. Oronzco, J. Phys. Chem., 97, 4386 (1993).

37. J.-L. Rivail and D. Rinaldi, Chem. Phys., 233 (1976).

38. J.-L. Rivail and B. Terryn, J. Chim. Phys., 79, 1 (1982).

39. C. J. Cramer and D. G. Truhlar, J. Am. Chem. Soc., 115, 8810 (1993).

40. B. Y. Simkin and I. E. Sheykhet, Quantum Chemical and Statistical Theory of Solutions. Computational Methods and Their Application, Moscow, Khimiya, 1989.

41. R. Bonaccorsi, R. Cammi, and J. Tomasi, J. Comp. Chem., 12, 301 (1991).

42. G. Alagona and C. Ghio, J. Mol. Structr. (Theochem), 256, 187 (1992).

43. R. Montagnani, J. Tomasi, and I. Demetropoulos, Mol. Eng., 2, 123 (1992).

44. N. L. Allinger, Am. Chem. Soc., 99, 8127 (1977).

45. L. Dosen-Micovic and N. L. Allinger, Tetrahedron, 34, 3385 (1978).
46. L. Dosen-Micovic, D. Jeremic, and N. L. Allinger, J. Am. Chem. Soc., 105, 1716 (1983).

47. D. M. Soumpasis, Proc. Natl. Acad. Sci. USA, 81, 5116 (1984).

48. D. M. Soumpasis, A. Garscia, R. Klement, and T. Jovin, In Theoretical Biochemistry \& Molecular Biophysics, D. L. Beveridge and R. Lavery, Eds., Adenine Press, Guilderland, NY, 1990, p. 343.

49. V. Fritsch and E. Westhof, J. Am. Chem. Soc., 113, 8271 (1991).

50. V. Fritsch, G. Ravishanker, D. L. Beveridge, and E. Westhof, Biopolymers, 33, 1537 (1993).

51. R. Lavery, H. Sklenar, K. Zakrzewska, and B. Pullman, J. Biomol. Struct. Dyn., 3, 989 (1986).

52. B. R. Brooks, R. E. Bruccoleri, B. D. Olafson, D. J. States, S Swaminathan, and M. Karplus, I. Comp. Chem., 4, 187 (1982).

53. R. J. Zauhar, J. Comp. Chem., 12, 575 (1991).

54. D. L. Beveridge and G. W. Schnuelle, J. Phys. Chem., 78, 2064 (1974)

55. D. L. Beveridge and G. W. Schnuelle, J. Phys. Chem., 79, 2562 (1975).

56. G. W. Schnuelle, S. Swaminathan, and D. L. Beveridge, Theoret. Chim. Acta, 48, 17 (1978).

57. K. Sharp, J. Comp. Chem., 12, 454 (1991).

58. C. Still, A. Tempczyk, R. C. Hawley, and T. Hendrickson, J. Am. Chem. Soc., 112, 6127 (1990).

59. W. Hassel, T. Hendrickson, and W. C. Still, Tetrahedron Comp. Method., 1, 103 (1988).

60. F. Mohamadi, N. G. J. Richards, W. C. Guida, R. Liscamp, M. Lipton, C. Caufield, G. Chang, T. Hendrickson, and W. C. Still, J. Comp. Chem., 11, 440 (1990).

61. P. Auffinger and G. Wipff, J. Am. Chem. Soc., 113, 5976 (1991).

62. P. Guilbaud, A. Varnek, and G. Wipff, J. Am. Chem. Soc., 115, 8298 (1993).

63. L. Troxler and G. Wipff, J. Am. Chem. Soc., 116, 1468 (1994).

64. P. Claverie, In Intermolecular Interactions: From Diatomics to Biopolymers, B. Pullman, Ed., Wiley: Chichester, 1978, p. 69.

65. M. K. Gilson, M. E. Davis, B. A. Luty, and J. A. McCammon, J. Phys. Chem., 97, 3591 (1993).

66. J. L. Pascual-Ahuir, E. Silla, J. Tomasi, and R. Bonaccorsi, J. Comp. Chem., 8, 778 (1987).

67. A. A. Rashin and K. Namboodiri, J. Phys. Chem., 91, 6003 (1987).

68. A. A. Varnek and A. S. Glebov, MM2IS Program Package, Mendeleev Institute of Chemical Technology, Moscow, 1991.

69. A. A. Varnek, A.S. Glebov, O. M. Petrukhin, and R. P. Ozerov, Koord. Khimiya (Coord. Chemistry, Rus.), 15, 600 (1989).

70. N. Gresh, P. Claverie, and B. Pullman, Int. J. Quant. Chem., 13, 243 (1979).

71. J. Caillet and P. Claverie, Acta Crystallogr., Sect. A, 31, 448 (1975).

72. R. J. M. Lefevre, Adv. Phys. Org. Chem., 3, 1 (1965). 
73. A. A. Varnek, A. S. Glebov, O. M. Petrukhin, and R. P. Ozerov, Koord. Khimiya (Coord. Chemistry, Rus.), 15, 740 (1989).

74. A. A. Varnek, A. Maya, D. Landini, A. Gamba, G. Morosi, and G. Podda, J. Phys. Org. Chem., 6, 113 (1993).

75. A. A. Varnek, G. Morosi, and A. Gamba, J. Phys. Org. Chem., 5, 109 (1992).

76. A. A. Varnek, J. E. ten Elshof, A. S. Glebov, V. P. Solov'ev, V. E. Baulin, and E. N. Tsvetkov, J. Mol. Structr. 271, 311 (1992).

77. A. Y. Tsivadze, A. A. Varnek, and V. E. Khutorsky, Coordination Compounds of Metals with Crown-Ligands (in Russian), Nauka Publishing House, Moscow, 1991.

78. R. Bonaccorsi and R. Cammi, MGPISA, ICQEM, Pisa, Italy.

79. Y. Marcus, Ion Solvation, Wiley, Chichester, 1985.

80. E. Engler and G. Wipff, MD_DRAW. A Program of Graphical Representation of Molecular Trajectories, Université Louis Pasteur, Strasbourg, 1992.

81. F. A. Momany, J. Phys. Chem., 82, 592 (1978).

82. MOPAC-5. QCPE Program No. 589 (1990).

83. M. A. Connolly, J. Appl. Crystallogr., 16, 548 (1983).

84. M. Billeter, A. E. Howard, I. D. Kuntz, and P. A. Kollman, J. Am. Chem. Soc., 110, 8385 (1988).

85. W. F. van Gunsteren and H. J. C. Berendsen, Groningen Molecular Simulation (GROMOS) Library Manual, Biomos, Groningen, 1987.

86. A. A. Varnek, A. S. Glebov, and D. Feil, GROMOS-MST program (1992).

87. J. Hermans, H. J. C. Berendsen, W. F. van Gunsteren, and J. P. M. Potsma, Bicpolymers, 23, 1513 (1984).

88. G. Wipff and L. Troxler, In Computational Approaches in Supramolecular Chemistry, G. Wipff, Ed., Kluwer Academic Publisher, Dordrecht, 1994, p. 319.

89. P. Aufinger and G. Wipff, J. Am. Chem. Soc., 113, 5976 (1991).

90. W. L. Jorgensen, J. Chandrasekhar, and J. D. Madura, J. Chem. Phys., 79, 926 (1983).

91. D. A. Pearlman, D. A. Case, J. C. Cadwell, G. L. Seibel, U.
C. Singh, P. Weiner, and P. A. Kollman, AMBER4, University of California, San Francisco, 1991.

92. S. J. Weiner, P. A. Kollman, D. T. Nguyen, and D. A. Case, J. Comp. Chem., 7, 230 (1986).

93. Handbook on Chemistry (in Russian), Khimiya Publishing House, Moscow, 1972.

94. P. Seiler, M. Dobler, and J. D. Dunitz, Acta Crystallogr. B, 30, 2744 (1974).

95. M. A. Bush and M. R. Truter, J. Chem. Soc. B, 1140 (1971).

96. M. Dobler, J. D. Dunitz, and P. Seiler, Acta Crystallogr. B, 30, 2741 (1974).

97. E. Maverick, P. Seiler, and J. D. Dunitz, Acta Crystallogr. B, 36, 615 (1980)

98. G. Ranghino, S. Romano, J.-M. Lehn, and G. Wipff, I. Am. Chem. Soc., 107, 7873 (1985).

99. L. Troxler and G. Wipff, J. Am. Chem. Soc., 116, 1468 (1994).

100. P. I. Nagy, W. J. Dunn III, G. Alagona, and C. Ghio, J. Am. Chem. Soc., 113, 6719 (1991).

101. P. I. Nagy, W. J. Dunn III, G. Alagona, and C. Ghio, J. Am. Chem. Soc., 114, 4752 (1992).

102. B. H. Besler, K. M. Merz, and P. A. Kollman, J. Comp. Chem., 11, 431 (1990).

103. R. Zauhar, private communication.

104. F. M. Floris, J. Tomasi, and J. L. P. Ahuir, J. Comp. Chem., 12, 784 (1991)

105. M. Oronzco, W. L. Jorgensen, and F. J. Luque, J. Comp. Chem., 14, 1498 (1993).

106. T. Simonson and A. T. Brünger, J. Phys. Chem., 98, 4683 (1994).

107. T. P. Straatsma and J. A. McCammon, J. Chem. Phys., 91, 3631 (1989).

108. A. Pullman, In Quantum Theory of Chemical Reactions, R. Daudel, Ed., Reidel, Amsterdam, 1980, p. 1.

109. B. Pullman, S. Miertus, and D. Perahia, Theor. Chin. Acta, 50, 317 (1979).

110. A. Pullman and D. Perahia, Theor. Chim. Acta, 48, 29 (1978).

111. F. T. H. Leuwerink, S. Harkema, W. J. Briels, and D. Feil, J. Comp. Chem., 14, 899 (1993). 